\begin{tabular}{|c|c|c|}
\hline 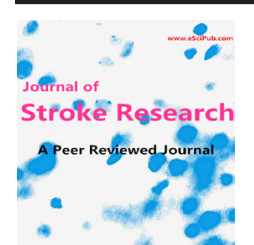 & $\begin{array}{c}\text { Journal of Stroke Research } \\
\text { (JSR) }\end{array}$ & 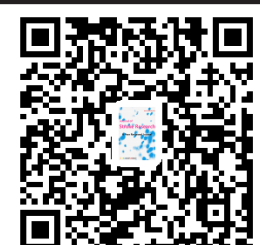 \\
\hline
\end{tabular}

\title{
Histopathological Changes Of Cerebral Vessels Secondary To Drug
}

\section{Abuse}

Zogopoulos Panagiotis, Theocharis Stamatios, Kotakidis Nikolaos, Patsouris Efstratios, Agapitos Emmanouel

First Department of Pathology, Medical School, National and Kapodistrian University of Athens, Greece

\section{ABSTRACT}

Drug abuse has been associated with a number of cerebrovascular adverse effects, such as hemorrhagic and ischemic stroke. Heroin, cocaine and amphetamines are the most commonly abused substances but a systematic pathology study of their effects on human cerebral vessels has not been conducted to date. We present a post-mortem, comparative, pathology study on the brains of drug addicts and healthy controls. The aim of this study was to determine the pathological changes of the vascular lumen, vascular wall and perivascular areas observed in the post-mortem brain of drug addicts compared to those of nondrug addicts healthy controls.

\section{Keywords:}

drug abuse, vascular changes, brain, pathology

${ }^{*}$ Correspondence to Author:

Zogopoulos Panagiotis

69 Vosporou str., Athens, Greece

P.O. Box: 10444

Tel.: +306976053555

E-mail: p.zogopoulos @ yahoo.com

How to cite this article:

Zogopoulos Panagiotis, Theocharis Stamatios, Kotakidis Nikolaos, Patsouris Efstratios, Agapitos Emmanouel. Histopathological Changes Of Cerebral Vessels Secondary To Drug Abuse. Journal of Stroke Research, 2016,1(1): 0013-0017.

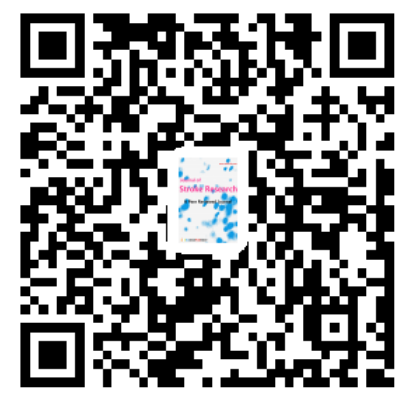

Accepted 24 August 2016; published 24 August 2016.

\section{eSciencePublisher}

eSciPub LLC, Houston, TX USA. Website: http://escipub.com/ 


\section{Introduction}

Drug abuse, a severe socioeconomic problem of modern societies, has evolved into a plague over the last decades. Heroin, cocaine, amphetamines and cannabis are the most commonly abused substances but other chemical compounds such as "ecstasy" and lysergic acid diethylamide (LSD) are also widely abused.

Since drug addicts rarely use sterile tools (e.g. syringes) and abuse multiple substances (often contaminated by foreign particles), infections as well as systemic inflammatory reactions can be seen, affecting among others the cerebral vessels. Due to the multiplicity of substance abuse, it is rather difficult to associate particular histopathological changes to a certain substance. Instead, these changes can be attributed to drug abuse as a whole.

\section{Materials and Methods}

A post-mortem study on the cerebral vessels of drug addicts and healthy controls was conducted and the findings among the two groups were compared. Patients with neurological disorders not related to drug abuse (trauma, stroke, epilepsy etc.) and/or vascular diseases (hypertension, coronary artery disease, diabetes mellitus etc.) were excluded from the study. Sixty five human brains (48 drug addicts and 17 healthy controls of similar age) were examined. The aim of our study was to determine the pathological changes of the vascular lumen, vessel wall and perivascular areas in the post-mortem brains of drug addicts and compare them with non-drug addicts healthy controls. Areas studied include the cerebral cortex, the cerebellum (both nuclei and white matter), basal ganglia, the hippocampal formation and the brainstem (pons). Light microscope was utilized to study the anatomic specimens which had been previously dyed with hematoxylin eosin. Toxicology analysis was performed to determine the substance used in each case. Statistical analysis was conducted using Student's t test and statistical significance was set to $p<0.05$.

\section{Results}

Vascular lumen findings

Pathological changes observed at the vascular lu- men of drug addicts' cerebral vessels include:

- Platelet aggregation

- Vascular lumen thrombosis

- Lymphocyte adhesion to the endothelial wall

- Atherosclerotic changes causing vascular lumen stenosis

In particular, platelet aggregation, vascular lumen thrombosis and atherosclerotic changes were observed in a few samples, but these findings were not statistically significant, in any of the brain areas examined. On the contrary, lymphocyte adhesion to the endothelial wall of drug addicts' cerebral vessels was statistically significantly observed in all areas examined (Fig. 1) and specifically: the frontal cortex $(p<0.05)$, the cerebellar cortex $(p<0.05)$ and dentate nucleus $(p<0.05)$, the globus pallidus $(p<0.05)$, the hippocampus $(p<0.01)$ and pons $(p<0.01)$.

Vascular wall findings

Pathological changes observed at the vascular wall of drug addicts' cerebral vessels include:

- Fibrinoid vasculitis - necrosis

- Transmural infiltration by inflammatory cells

- Pigment deposition

- Swelling of the vascular wall

- Fibrinoid degeneration of the vascular wall

- Degenerative - atherosclerotic lesions

In particular, fibrinoid vasculitis/necrosis, pigment deposition, swelling and degenerative/atherosclerotic lesions of the vascular wall were observed in a small number of samples, but these findings were not statistically significant, in any of the brain areas examined. On the contrary, transmural infiltration by inflammatory cells was statistically significant in all areas of the drug addicts' brains examined (Fig. 2) and specifically: the frontal cortex $(p<0.05)$, the cerebellar cortex $(p<0.05)$ and dentate nucleus $(p<0.05)$, the globus pallidus $(p<0.05)$, the hippocampus $(p<0.05)$ and pons $(p<0.05)$. Furthermore, fibrinoid degeneration of the vascular wall was statistically significantly observed at the globus pallidus $(p<0.05)$, hippocampus $(p<0.05)$ and pons $(p<0.01)$, but not at the frontal 
cortex or the cerebellum (cortex or dentate nucleus).

Perivascular findings

Pathological changes observed at the cerebral perivascular areas of drug addicts include:

- Perivascular infiltration by inflammatory cells

- Perivascular edema

- Hemorrhage

In particular, there was no statistically significant difference regarding any of the above changes at the frontal cortex, the cerebellum (cortex and dentate nucleus) and pons between drug addicts and control group. On the contrary, perivascular infiltration by inflammatory cells (Fig. 3 \& 4) was statistically significantly observed at the globus pallidus $(p<0.05)$, while perivascular edema $(p<0.01)$ and hemorrhage $(p<0.01)$ were statistically significant at the hippocampus of drug addicts compared to the control group.

\section{Discussion}

Drugs are abused primarily due to their psychotropic properties but most of them also exhibit systemic and adverse effects in the central and peripheral nervous sytem. Neurological complications can occur during acute toxicity, after short-term abstention or as a consequence of long-term abuse. Moreover, various contaminants and additives, such as procainamide, quinine, strychnine and several sugars found in drugs can cause allergic reactions and have their own adverse effects (1).

Extensive alterations of the vascular basal membrane have been observed in the cerebral cortex of drug abusers. For example, in the gray matter of the frontal, parietal and occipital lobe the number of vessels with strong immunoreactivity for collagen type IV is significantly reduced, while the number of vessels with mild or moderate immunoreactivity has been found increased compared with the control group (2). The total number (density) of vessels did not show significant changes in the temporal, parietal or occipital cortex, however, in the frontal cortex a significant decrease in the density of vessels was found (2). This finding could suggest a possible hypoperfusion of the frontal lobes, thus explaining the behavioral changes and motor impairment which are commonly seen in drug abusers. The number (density) of vessels in the white matter of abusers were not significantly different from the control group (2).

The first report of cerebral hemorrhage associated with drug abuse was in 1945 for amphetamines (3) and in 1977 for cocaine (4). Numerous clinical observations and case reports in the literature suggest that cocaine abuse can lead to severe complications regarding cerebral vessels. After the introduction of cocaine alkaloid "crack" in the early 1980's the incidence of cocaine associated strokes has increased dramatically. Apart from few cases of vasculitis, the etiology of cocaine associated stroke (ischemic and hemorrhagic strokes, subarachnoid hemorrhage, intracerebral hemorrhage and cerebral ischemia) still remains largely unknown. Proposed mechanisms (other than the drug-induced vasospasm) include hemostasis disorder, platelet dysfunction and reduction of cerebral blood flow. Cerebral vasculitis has been associated with amphetamines (5), ephedrine (6), heroin (7), phenylpropanolamine (8), lysergic acid diethylamide (LSD) but also with multiple substance abuse (9). Particularly, cocaine abuse has also been associated with other types of vasculitis such as necrotizing vasculitis in Schoenlein-Henoch purpura (10), Churg-Strauss vasculitis (11) and Fusarium endophthalmitis (12).

Masson trichrome stain has revealed extensive vacuolar degeneration in the cerebral white matter of patients with heroin-induced spongiform leukoengephalopathy (HSLE)(13). Vacuoles were arranged around gray matter capillaries and arterioles, while no vacuoles were found around cerebral blood vessels of the control group (13). Immunohistochemistry studies revealed that CD34 positive cells were seldom found around blood vessels of frontal lobe gray matter, cerebellar white matter and corpus callosum of HSLE patients (13). However, one type of CD34 positive cells was found in the vascular wall of control group subjects $(P<0.001)$. The reduction or even loss of CD34 positive cells indicates endothelial cell damage in the cerebral vessels of HSLE patients (13).

Angiographic and histological studies in humans and experimental animals have shown that blood vessels of all diameters are involved in drug abuse vasculitis, but small vessel disease (small arterioles and capillaries) is prevalent (14-18). Since cerebral angiography and/or cerebral biopsy are necessary to establish diagnosis, the true incidence of drug abuse-associated vasculitis is unknown. 


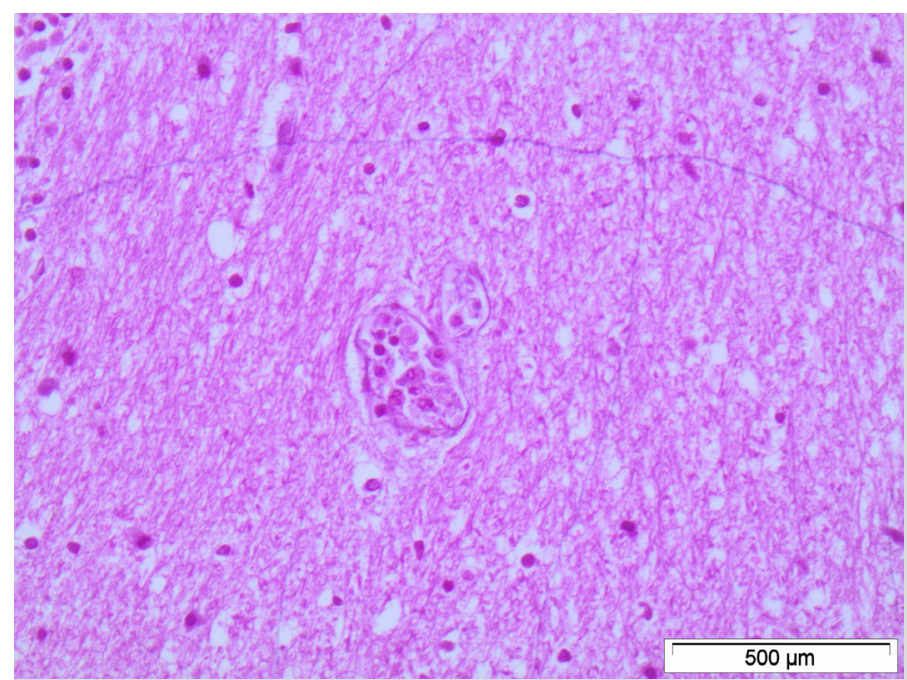

Figure 1: Inflammatory cells adhesion to vascular wall.

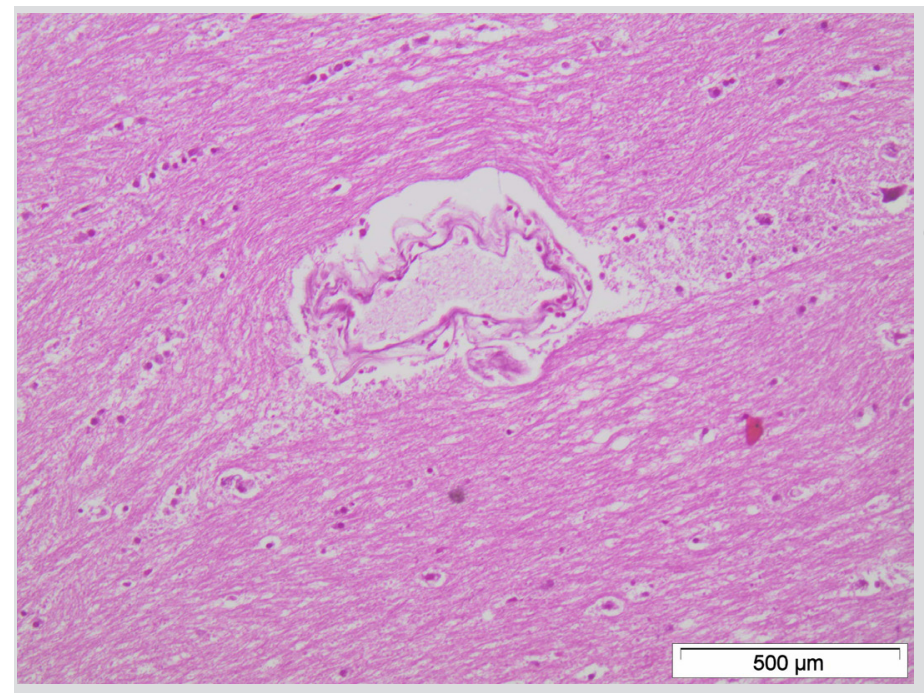

Figure 2: Vascular wall infiltration by inflammatory cells and perivascular edema.

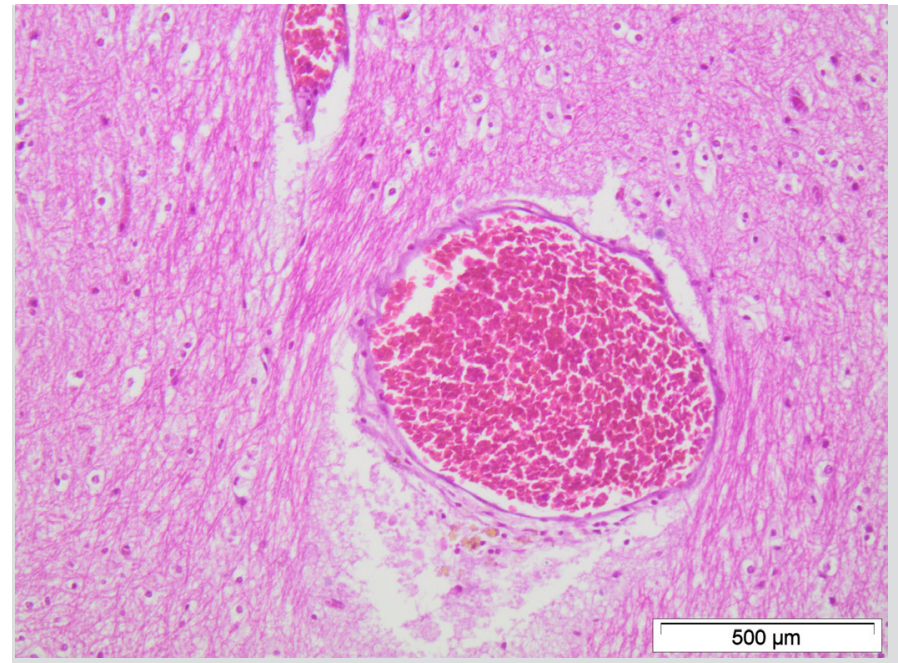

Figure 3: Perivascular infiltration by inflammatory cells and perivascular edema.

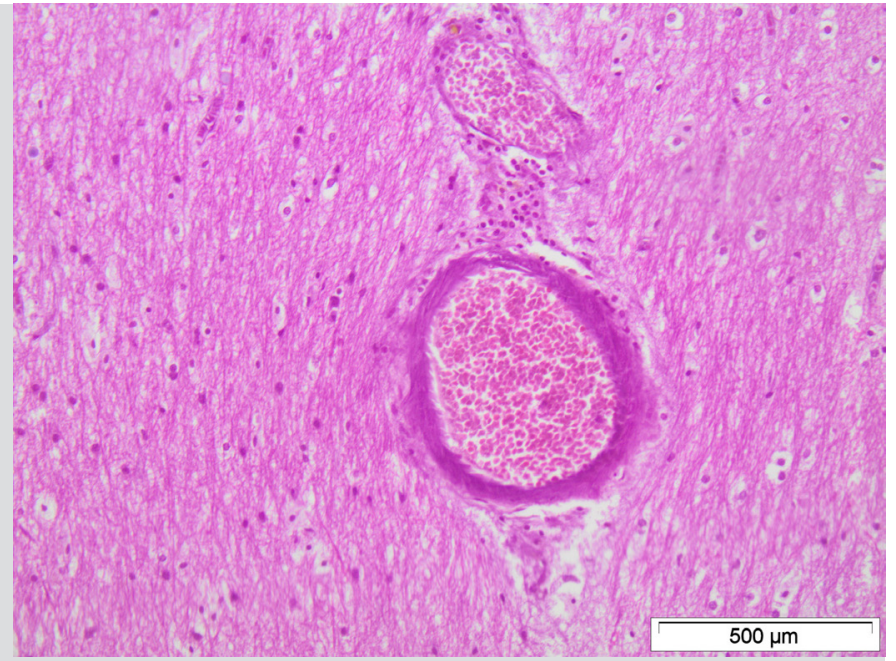

Figure 4: Vascular wall and perivascular infiltration by inflammatory cells and edema. 


\section{Conclusion}

Various cerebrovascular events (cerebral ischemia and infarction, subarachnoid hemorrhage, intracerebral hemorrhage etc.) in drug abusers have been reported (mostly as case reports) in the past. A comparative, systematic pathology study, however, had not been conducted. All the changes in vascular lumen, vascular wall and perivascular areas of drug abusers' brain reported here suggest a mechanism of neurological decline commonly observed in these patients and may propose a new therapeutic target for their treatment.

\section{Acknowledgements}

This study was conducted with the support of IKY Fellowships of Excellence for Postgraduate studies in Greece - Siemens Program.

\section{Conflict of Interest}

None to declare.

\section{References}

1. Gay G.R. Clinical management of acute and chronic cocaine poisoning. Am Emerg Med. 1982;11:562-572.

2. Büttner A., Kroehling C., Mall G., Penning R. and Weis $\mathrm{S}$. Alterations of the vascular basal lamina in the cerebral cortex in drug abuse: a combined morphometric and immunohistochemical investigation. Drug and Alcohol Dependence. 2005;79:63-70.

3. Gericke O.L. Suicide by ingestion of amphetamine sulphate. JAMA. 1945;128:1098-1099.

4. Brust J.C. and Richter R.W. Stroke associated with cocaine abuse? N Y State J Med. 1977;77:14731475.

5. Salanova V. and Taubner R. Intracerebral haemorrhage and vasculitis secondary to amphetamine use. Postgrad Med. 1984;60:429-430.

6. Wooten M.R., Khangune M.S. and Murphy M.J. Intracerebral haemorrhage and vasculitis related to ephedrine abuse. Ann Neurol. 1983;13:337340.

7. King J., Richards M. and Tress B. Cerebral arte- ritis associated with heroin abuse. Med $\mathrm{J}$ Aust. 1978;2:444-445.

8. Kase C.S., Foster T.E., Reed J.E., Spatz E.L. and Girgis G.N. Intracerebral haemorrhage and phenylpropanolamine. Neurology. 1987;37:399-404.

9. Citron BP, Halpern M, McCarron M, Lundberg GD, McCormick R, Pincus IJ, Tatter D and Haverback BJ. Necrotizing angiitis associated with drug abuse. N Engl J Med. 1970;283:1003-1011.

10. Chevalier X., Rostoker G., Larget-Piet B. and Gherardi R. Schoenlein-Henoch purpura with necrotizing vasculitis after cocaine snorting. Clin. Nephrol. 1995;43:348-349.

11. Orriols R., Munoz X., Ferrer J., Huget P. and Morell $F$. Cocaine-induced Churg-Strauss vasculitis. Eur. Respir. J. 1996;9:175-177.

12. Gabriele P. and Hutchins R.K. Fusarium endophthalmitis in an intravenous drug abuser. Am. J. Ophthalmol. 1996;122:119-121.

13. Yin J., Pan S.Y., Zhou L., Lu T.M., Luo Y.F. and Lu B.X. Pathological analysis of heroin spongiform leukoencephalopathy. J South Med Univ. 2007;27(6):881-886.

14. Halpern $M$ and Citron BP. Necrotizing angiitis associated with drug abuse. Am J Roentgenol Radium Ther Nucl Med. 1971;111:663-671.

15. Rumbaugh C.L., Bergeron R.T., Scanlan R.L., Teal J.S., Segall H.D., Fang H.C. and McCormick R. Cerebral vascular changes secondary to amphetamine abuse in the experimental animal. Radiology. 1971;101:345-351.

16. Rumbaugh C.L., Bergeron R.T., Fang H.C.L. and McCormack R. Cerebral angiographic changes in the drug abuse patient. Radiology. 1971;101:335344.

17. Rumbaugh $\mathrm{CL}$, Fang $\mathrm{HC}$, Higgins RE, Bergeron RT, Segall HD and Teal JS. Cerebral microvascular injury in experimental drug abuse. Invest Radiol. 1976;11:282-294.

18. Kaye B.R. and Fainstat M. Cerebral vasculitis associated with cocaine abuse. J.A.M.A. 1987;258:2104-2106.

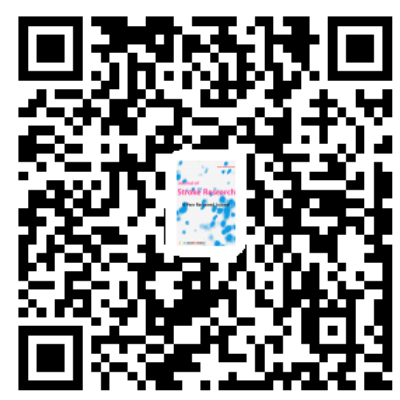

